\title{
Assessment of Burden of COPD tool: evidence not perception
}

\author{
To the Editor:
}

We thank Houben-Wilke et al. [1] for their comments on the Assessment of Burden of COPD (ABC) tool. They raised questions about the purpose of the $\mathrm{ABC}$ tool. The aims of $\mathrm{ABC}$ were clarified before its development, in the study protocol [2]. The $\mathrm{ABC}$ tool is multifaceted and was developed to measure burden of disease, facilitating shared decision making and formulation of a personal treatment plan [2-5]. All of these purposes relate to increasing self-management and stimulating behavioural change, which are, most often, important components of individual treatment plans. The goal of most treatment plans is to increase health status. Health status was therefore the primary outcome of our study [2-5].

We agree with HoubEN-Wilke et al. [1] that comorbidities play a major role in the burden and management of COPD. Both in the study protocol [2] and the article describing the effectiveness study [5], we describe that comorbidity is part of this tool.

The $\mathrm{ABC}$ tool was developed by a nationwide, multidisciplinary working group and has been proven to have an impact on care [2-5]. The $\mathrm{ABC}$ tool goes beyond assessing health status. It provides a visualisation of outcomes and treatment advice, based on national guidelines, to help patients and healthcare providers set a personal goal and make an individual treatment plan. The added value of the $\mathrm{ABC}$ tool is the combination of these characteristics in one simple tool. In our qualitative evaluation of the $\mathrm{ABC}$ tool [4], we described that patients and healthcare providers evaluate the $\mathrm{ABC}$ tool as a very useful instrument that has important added value in daily care. Houben-WiLke et al. [1] suggest that the simplicity of the balloons masks the lack of scientific and clinical evidence. However, we believe that simplicity is, if anything, the strength of the tool, confirmed by patients and healthcare providers participating in the trial [4], and by the significant positive results of the trial: the use of the ABC tool significantly increased health status and quality of care [5]. The ease of use of the ABC tool makes it attractive and practical as an instrument for shared decision making. The balloon diagram can be used as a starting point for good communication between the patient and healthcare provider, which is requisite for personalised care [3].

In addition, Houben-Wilke et al. [1] raised some issues regarding the methodology and interpretation of the study results. Regarding potential baseline differences, we have indeed not tested for between-group statistical differences at baseline. This is in line with the international CONSORT (Consolidated Standards of Reporting Trials) statement, which states that it is "illogical to test for baseline differences" in randomised trials [6]. The appropriate method is to adjust the analysis for possible differences at baseline, as we did in our study [5]. The baseline difference of the outcome at hand was, in fact, adjusted for in two different ways, one corresponding to including the baseline as repeated measure and one corresponding to including the baseline as covariate (for technical details, see the articles by Liu et al. [7] and vaN BREUKeLEN [8]). Both methods led to very similar conclusions.

Houben-Wilke et al. [1] suggest that although there was a statistically significant difference in patients with a clinical relevant improvement in health status, the mean difference in health status score did not change. In figure 4 of the original article of our study [5] (reproduced here as figure 1), we visualised the change and differences between the intervention and control groups. The differences were indeed statistically significant at 12 and 18 months ( $\mathrm{p}=0.004$ and $\mathrm{p}=0.008$, respectively), as shown in table 2 of the original article [5]. For details of our analyses, see the Statistical Analyses in the Methods section and see the legend of table 2 in our original article [5].

@ERSpublications

ABC tool affects HRQoL and quality of primary/secondary COPD care but has not been tested in tertiary settings http://ow.ly/HZYF30cmTsx

Cite this article as: Slok AHM, Kotz D, van Breukelen G, et al. Assessment of Burden of COPD tool: evidence not perception. Eur Respir J 2017; 50: 1700756 [https://doi.org/10.1183/13993003.00756-2017]. 


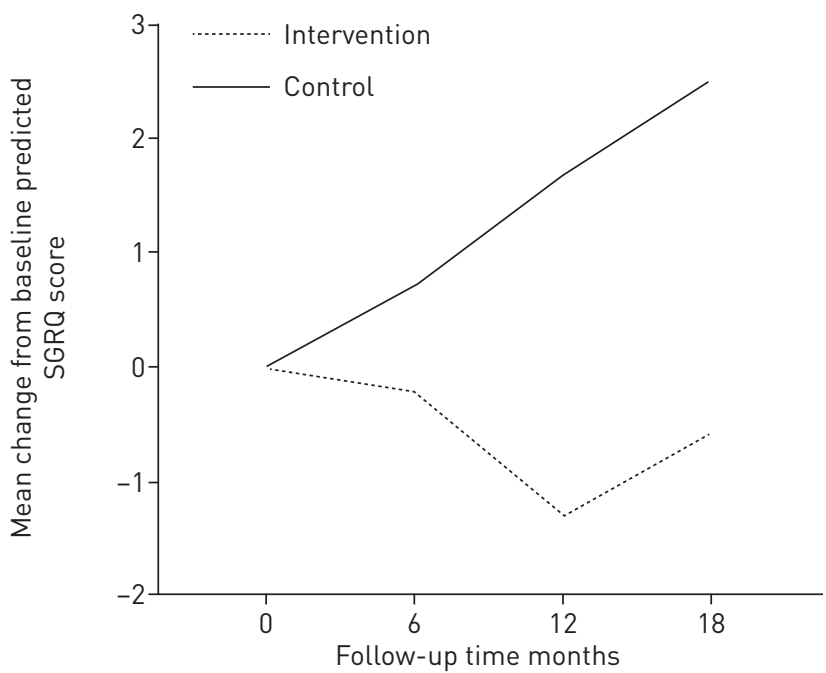

FIGURE 1 Mean change in predicted St George's Respiratory Questionnaire (SGRQ) total scores at 6-, 12- and 18-month follow-up compared with baseline, with a higher score indicating worse quality of life. Reproduced and modified from [5] with permission from the publisher.

Houben-Wilke et al. [1] correctly state that we did not report a minimal clinically important difference for the Patient Assessment of Chronic Illness Care (PACIC) in our study as this cut-off point is not available in the literature. The statistically significant improvement we found, however, is comparable to changes observed in other studies $[9,10]$.

We checked whether there is a correlation between a change in health status of patients and an improvement in perceived quality of care, as Houben-WILKE et al. [1] suggest. These post hoc analyses showed that there is indeed a significant correlation between the difference in the St George's Respiratory Questionnaire score between baseline and follow-up, and the difference in the decision support subdomain of PACIC ( $\mathrm{r}=-0.160, \mathrm{p}=0.008$ ). This indicates that the improved health status might at least partially be related to an improved perceived healthcare. The underlying mechanisms of the effect cannot be fully clarified on the basis of data collected in this pragmatic, randomised controlled trial.

We might have had a case of "never the two worlds shall meet" from the moment we made the evidence-based decision not to use the instrument developed by Vercoulen and colleagues, the Nijmegen Clinical Screening Instrument [3]. We did so based on a well-documented appraisal of measurement properties of 21 available instruments [3]. On one thing we do agree, however: our ABC tool is simple to use. It was intended to be, it is supposed to be, and we have provided evidence that its use has an effect on quality of primary and secondary care [2-5]. HouBEN-WiLKe et al. [1] are all affiliated with tertiary referral centres for home rehabilitation. We have not tested our instrument in that setting but we invite them to do so. Finally, we do admire the valuable work of these highly specialist centres, in their settings with their specific group of patients.

Annerika H.M. Slok ${ }^{1}$, Daniel Kotz ${ }^{1,2}$, Gerard van Breukelen ${ }^{3}$, Niels H. Chavannes ${ }^{4}$,

Maureen P.M.H. Rutten-van Mölken ${ }^{5}$, Huib A.M. Kerstjens ${ }^{6}$, Thys van der Molen ${ }^{7}$, Guus M. Asijee ${ }^{1,8}$, P.N. Richard Dekhuijzen', Sebastiaan Holverda ${ }^{10}$, Philippe L. Salomé ${ }^{11}$, Lucas M.A. Goossens ${ }^{5}$, Mascha Twellaar ${ }^{1}$, Johannes C.C.M. in ' $t$ Veen ${ }^{12}$ and Onno C.P. van Schayck ${ }^{1}$

${ }^{1}$ Dept of Family Medicine, Care and Public Health Research Institutie (CAPHRI), Maastricht University, Maastricht, The Netherlands. ${ }^{2}$ Institute of General Practice, Addiction Research and Clinical Epidemiology Unit, Medical Faculty of the Heinrich Heine University Düsseldorf, Dusseldorf, Germany. ${ }^{3}$ Dept of Methodology and Statistics, CAPHRI, Maastricht University, Maastricht, The Netherlands. ${ }^{4}$ Dept of Public Health and Primary Care, Leiden University Medical Centre, Leiden, The Netherlands. ${ }^{5}$ Institute for Health Policy and Management/Institute for Medical Technology Assessment, Erasmus University Rotterdam, Rotterdam, The Netherlands. ${ }^{\circ}$ Dept of Pulmonary Diseases, Groningen Research Institute for Asthma and COPD (GRIAC), University of Groningen, University Medical Centre Groningen, Groningen, The Netherlands. ${ }^{7}$ Dept of General Practice, University of Groningen, University Medical Centre Groningen, Groningen, The Netherlands. ${ }^{8}$ Foundation PICASSO for COPD, Alkmaar, The Netherlands. ${ }^{9}$ Dept of Pulmonary Diseases, Radboud University Medical Centre, Nijmegen, The Netherlands. ${ }^{10}$ Lung Foundation Netherlands, Amersfoort, The Netherlands. ${ }^{11}$ Huisartsencoöperatie PreventZorg, Bilthoven, The Netherlands. ${ }^{12}$ Dept of Pulmonology, and SZT Centre of Excellence for Asthma and COPD, Franciscus Gasthuis and Vlietland, Rotterdam, The Netherlands.

Correspondence: Annerika H.M. Slok, Dept of Family Medicine, CAPHRI, Maastricht University, PO Box 616, Maastricht, 6200MD, The Netherlands.

E-mail: annerika.slok@maastrichtuniversity.nl 
Received: April 112017 | Accepted: April 282017

Conflict of interest: Disclosures can be found alongside this article at erj.ersjournals.com

\section{References}

1 Houben-Wilke S, Franssen FM, Kok I, et al. Assessment of Burden of COPD (ABC) tool: perception versus evidence. Eur Respir J 2017; 49: 1602382.

2 Slok AHM, In 't Veen JCCM, Chavannes NH, et al. Effectiveness of the Assessment of Burden of Chronic Obstructive Pulmonary Disease $(\mathrm{ABC})$ tool: study protocol of a cluster randomised trial in primary and secondary care. BMC Pulm Med 2014; 14: 131.

3 Slok AHM, In 't Veen JCCM, Chavannes NH, et al. Development of the Assessment of Burden of COPD tool: an integrated tool to measure the burden of COPD. NPJ Prim Care Respir Med 2014; 24: 14021.

4 Slok AHM, Twellaar M, Jutbo L, et al. "To use or not to use": experiences of healthcare professionals and patients with the Assessment of Burden of COPD (ABC) tool. NPJ Prim Care Respir Med 2016; 26: 16074

5 Slok AHM, Kotz D, van Breukelen G, et al. Effectiveness of the Assessment of Burden of COPD (ABC) tool on health-related quality of life in patients with COPD: a cluster randomised controlled trial in primary and hospital care. BMJ Open 2016; 6: e011519.

6 Moher D, Hopewell S, Schulz KF, et al. CONSORT 2010 explanation and elaboration: updated guidelines for reporting parallel group randomised trials. J Clin Epidemiol 2010; 63: e1-37.

7 Liu GF, Lu K, Mogg R, et al. Should baseline be a covariate or dependent variable in analyses of change from baseline in clinical trials? Stat Med 2009; 28: 2509-2530.

8 van Breukelen GJ. ANCOVA versus change from baseline in nonrandomized studies: the difference. Multivariate Behav Res 2013; 48: 895-922.

9 Smidth M, Olesen F, Fenger-Gron M, et al. Patient-experienced effect of an active implementation of a disease management programme for COPD - a randomised trial. BMC Fam Prac 2013; 14: 147.

10 Cramm JM, Rutten-Van Molken MP, Nieboer AP. The potential for integrated care programmes to improve quality of care as assessed by patients with COPD: early results from a real-world implementation study in The Netherlands. Int J Integr Care 2012; 12: e191. 